\title{
Heart Rate Variability: On the Importance to Perform HRV Analysis in Subjects Affected from Muscular Dystrophy
}

\author{
Elio Conte ${ }^{1,2 *}$, Ken Ware ${ }^{3}$, Riccardo Marvulli1,4, Giancarlo Ianieri' ${ }^{1,4}$, Marisa Megna1,4, \\ Sergio Conte ${ }^{1}$, Leonardo Mendolicchio ${ }^{1}$, Enrico Pierangeli ${ }^{1}$ \\ ${ }^{1}$ School of Advanced International Studies on Applied Theoretical and Non Linear Methodologies of Physics, \\ Bari, Italy \\ ${ }^{2}$ Department of Basic Sciences, Neuroscience and Sense Organs, University of Bari Aldo Moro, Bari, Italy \\ ${ }^{3}$ The International Neuro Physics Functional Performance Institute, Energy Circuit, Robina, Australia \\ ${ }^{4}$ Department of Physical Medicine and Rehabilitation, University of Bari Aldo Moro, Bari, Italy \\ Email: ${ }^{*}$ elio.conte@fastwebnet.it
}

Received 12 May 2015; accepted 7 June 2015; published 10 June 2015

Copyright (C) 2015 by authors and Scientific Research Publishing Inc.

This work is licensed under the Creative Commons Attribution International License (CC BY).

http://creativecommons.org/licenses/by/4.0/

(c) (i) Open Access

\section{Abstract}

This paper discusses a case of muscular dystrophy on which it has been performed HRV analysis. The results that we obtain evidence that the subject delineates a net ANS dysfunction. All the basic parameters relating standard time and frequency domain of $\mathrm{HRV}$ analysis result profoundly altered. Examination by Poicaré plot evidences in particular that the subject has an atrial fibrillation. Non linear indexes ApEn and Samp En evidence the very high condition of risk of the subject. The merging indication is for the cardiologist and scholars in cardiovascular disease to perform always ANS investigation in subjects in case of muscular dystrophy. After the NPT treatment, the subject obtains a net improvement.

\section{Keywords}

ANS Dysfunction in Muscular Dystrophy, HRV Analysis, Atrial Fibrillation

This paper is a short communication that aims to report to specialists in cardiology and cardiovascular diseases the importance of performing HRV analysis in case of muscular dystrophy.

The basic problem is of interest from many years.

"Corresponding author.

How to cite this paper: Conte, E., Ware, K., Marvulli, R., Ianieri, G., Megna, M., Conte, S., Mendolicchio, L. and Pierangeli, E. (2015) Heart Rate Variability: On the Importance to Perform HRV Analysis in Subjects Affected from Muscular Dystrophy. World Journal of Cardiovascular Diseases, 5, 141-149. http://dx.doi.org/10.4236/wjcd.2015.56017 
The autonomic nervous system plays a crucial role in heart rate (HR) modulation. It is retained to be an ascertained result that a decrease in the variability of heart rhythm is a predictor of morbidity and mortality [1] [2]. Heart rate variability (HRV) analysis represents presently a valid method evaluating the activity of the autonomic nervous system, one of the most promising markers. The apparently easy derivation of this measure has led to its use becoming popular. The cardiologist has been provided with a seemingly simple tool both for research and clinical studies. Indices of heart rate variability provide a window onto autonomic modulation of the heart. HRV indices, determined in either the time or frequency domain, are closely related and reflect parasympathetic, mixed sympathetic, and parasympathetic and circadian rhythms. Depression of HRV has been observed in many clinical scenarios, including autonomic neuropathy, heart transplantation, congestive heart failure (CHF), myocardial infarction (MI), and other cardiac and non-cardiac diseases. Consequently, the HRV analysis has entered as standard methodology to be employed as a simple, inexpensive and non-invasive method that provides information on neurocardial integrity. In addition to standard methods based on the use of time linear indexes and Fats Fourier Transform in frequency domain (FFT), recently, we have introduced a new method, called CZF, to evaluate heart rhythm variability in old as well as in young subjects [3]-[5].

Facioscapulohumeral muscular dystrophy (FSHD) is the third most frequent form of muscular dystrophy. Myotonic dystrophy (MD) is the most frequent form of muscular dystrophy in adults. Alterations of the cardiac physiology are often present in this pathology and clinical manifestations include alteration of the conduction delay, rhythm irregularities, and myocardial disease. Electrocardiographic investigations indicate often disorders as dysfunctions in intraventricular and atrioventricular conduction, atrial fibrillation and ventricular arrhythmias. Histopathology data show fibrosis in the conduction system and in the sinoatrial node associated to myocyte hypertrophy [6] [7]. Rather recently a consistent number of investigations have been proposed on the overall function of the autonomic nervous system in patients with muscular dystrophy (MD). The first emerging indication is that the presence of autonomic neuropathy in such MD pathology is highly unlikely [8] [9]. However, such studies on autonomic modulation of HR in patients with MD result highly conflicting [10]-[14]. The aim of the present paper is to report on such our recent study that we have performed on a subject affected from Facioscapulohumeral Muscular Dystrophy. On this subject we have performed HRV analysis finding a profound ANS dysfunction in its modulating action on heart. Consequently, we retain that it may be of interest to suggest to cardiologists and scholar in cardiovascular diseases to perform HRV analysis in case of such pathology.

In detail, we have published the results of this study elsewhere [15] [16]. We have examined this subject under the neurological profile evidencing by SMR (sensory motor rhythm) analysis the serious starting condition of the subject and his improvement after a treatment that we have called NPT and that is due to one of us (KW). In the paper we have also documented the objective and clinical improvement of the subject after such NPT treatment. We have also performed the HRV analysis on this subject evidencing his net improvement.

Consequently, the reason of such our new paper is not to add new results but to reconsider the obtained results of the HRV analysis so that to support the thesis of a net involvement of the ANS in this pathology and, in particular, to submit this result to the consideration and evaluation of the cardiologists and encourage them to perform HRV analysis in such cases of pathology. This is the reason to add thus such further comment on a journal as WJCD that is particular devoted to scholars specialized in the study of the whole cardiovascular system.

We would not consider here all the details about the subject that was submitted to the treatment, the analysis that we performed and the results that were obtained. The reader may find all the details in [15] [16]. Only we will resume some results from these works and in accordance with the purpose of informing that relates the present short paper.

We arranged the experimental set up in the following manner. We used a computer interfaced device having eight channels, six devoted to EMG recording, one devoted the ECG and one to EEG. Obviously we are interested only to the ECG. We examined the subject at rest before the treatment and soon after the treatment. The ECG was sampled at a frequency of $960 \mathrm{~Hz}$ and after recording, we extracted the R-R time intervals of the QRS complexes by a suitable algorithm so to have the final R-R time series and thus the tachograms were suitable for the HRV analysis. On these time series, we performed first of all the standard analysis as outlined by the ESC task Force [17]. They are the time linear indexes and the FFT or DFT that, as it is well known, is able to give, in addition to the PSD (total Power Spectral Density), substantially three bands, the VLF that relates thermoregulation, hormonal activity and rennin-angiotensin system and, generally speaking, the circadian rhythms, the LF band that relates substantially the orthosympathetic activity (really mixed with parasympathetic components) 
and the finally the HF band that relates parasympathetic activity and respiration. Soon after we used the CZF method that we indicated previously [3]-[5], The application of this method is so important since, using the same previously VLF, LF, HF bands, estimates this time the variability of the heart rhythm, analyzing variability in heart rhythm between adjacent heart beats and thus giving accurate and quantitative information on the heart modulating ANS activity inducing heart rhythm variability step by step. Soon after we examined also the histograms distribution of the obtained R-R intervals and HR. Finally we studied the Poincaré plot. This is an important method since it enables to obtain a phase space representation and distribution of the R-R intervals by plotting $R R_{n} / R_{n+1}$ that is to say the previous against the subsequent $R-R$ time intervals and giving in addition some basic indexes of evaluation that are called SD1, SD2, ellipse are and centroid [17]. Finally, we examined also non linear indexes that are the Approximate Entropy (ApEn), the Sample Entropy (SampEn) and the detrended fluctuation analysis indexes (DFA). The reason to examine HRV by such indexes is that R-R signal is substantially a non periodic, non stationary and mainly a non linear signal arising from a lot of modulating components. The arising time dynamics is very complex, possibly chaotic and fractal. A reduction of the previously mentioned indexes is indicative that heart rhythm is reduced exposing the subject to those conditions of risk of morbidity and mortality [1] [2] that we mentioned previously. Consequently, they give an accurate prognostic indication. The fractal regime in HR is signed in DFA analysis by two indexes, called alpha 1 and alpha 2, and they are indicative, respectively, of the short and long range correlations that the R-R values maintain in heart rhythm.

In conclusion, such kind of HRV analysis is able to identify from one hand the possible ANS dysfunction and, on the other hand, to identify and to classify the possible presence of one of the eight fundamental arrhythmias.

The examined subject evidenced at rest and before treatment a profound ANS alteration. The tachogram clearly evidenced the presence of a strong arrhythmia. All the time linear indexes resulted profoundly out of the normal range. The performed Fourier analysis evidenced a total Power Spectral Desity (PSD) out of the normal range as well as VLF, LF and HF bands indicated a large ANS dysfunction. The CZF evidenced in particular a strong activity of orthosympathetic activity. The histograms realized on R-R and on HR intervals revealed a serous deviation from a normal distribution. In particular, the analysis of the Poincaré Plot revealed with its distribution and indexes the ANS dysfunction and the presence of atrial fibrillation. The non linear indexes ApEn and SampEn gave very discouraging results, largely distant from the normal values and indicating the high level of risk of the subject. Only the DFA analysis gave results not so distant from normal values.

The results of this HRV analysis clearly supports the thesis that ANS dysfunction is identified in HRV of subjects affected from muscular dystrophy and encourages cardiologist and scholars in cardiovascular diseases to take into account also HRV analysis in such case of pathology.

There is a final result. The NPT treatment not only gave encouraging results of improvement under the neuromuscular profile [15] [16] as well as under the clinical and objective evaluation of the conditions of the subject but also induced a net improvement of the ANS dysfunction previously observed. In fact, all the indexes previously examined in the HRV analysis re-entered in a satisfactory manner in the normal range.

We add here an Appendix A containing the figures that of course we have reported in [16] and we give the results of our HRV analysis performed on the subject at rest before and after the treatment. We represent them here again only for convenience of the reader.

Figure 1: It gives the elaborated tachogram and our DFT results for the subject at rest before of the treatment. We observe a profound arrhythmia in the tachogram and profoundly altered values in the three VLF, LF, HF bands of the DFT analysis. In Figure 2 we have the obtained tile linear indexes. They are all out of the range with respect to the normal values established in the Task Force in [1] [2]. In Figure 3 and Figure 4, we have the results of application of our CZF method for analysis of variability of Heart Rhythm. We have discussed in detail this method in [3]-[5]. We re-outline here that this method enables us to explore variability in heart rhythm between subsequent beats (one time in absolute value, thus estimating the entity of the variation and one time without absolute value and thus evaluating acceleration and deceleration of the beat). Both such information is important in order to inspect the behaviour of heart rhythm. In absolute value we estimate the entity of the induced ANS modulation between subsequent beats; without absolute value we inspect the alternans that must be presented in heart rhythm during the ANS modulation. In such figures in red the graph gives indication of the corresponding heart rhythm behaviour beat after beat and we verify the profound arrhythmia of the subject. By the CZF we also estimate the obtained values in VLF, LF, HF bands and we find the profound alterations with respect to the normal values, given in [3]-[5]. In Figure 5 and Figure 6 we have HR and R-R histogram distribution. In normal conditions we have a rather normal statistical distribution. The direct inspection gives instead 

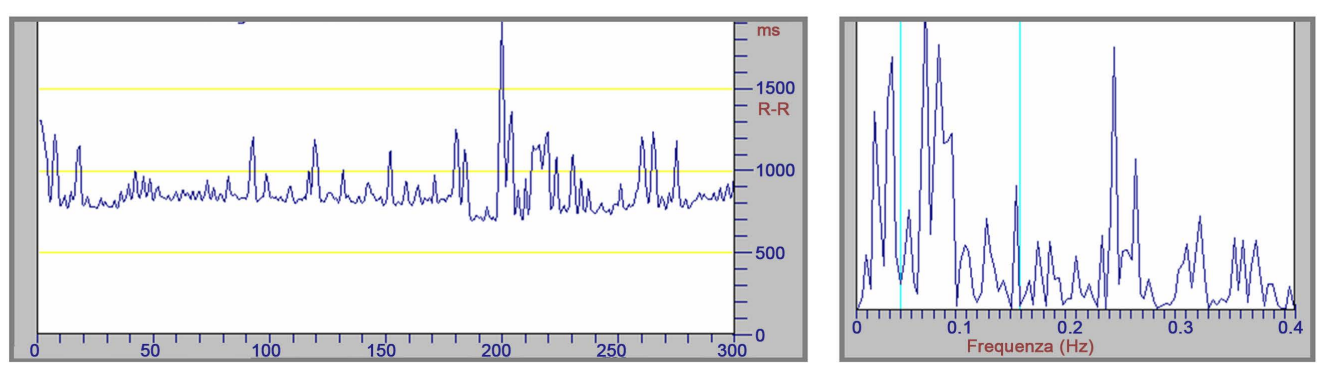

Figure 1. Tachogram obtained after ECG recording PSD Spectrum $\left(\mathrm{msec}^{2} / \mathrm{Hz}\right)$ with three delineated bands, VLF, LF and HF.

\begin{tabular}{|c|c|c|c|c|c|}
\hline \multicolumn{6}{|c|}{ All Data } \\
\hline & RRI & HR & & RRI & HR \\
\hline Maximume & $896.66 \mathrm{~ms}$ & $180.00 \mathrm{bpm}$ & InHAV: & 10.6457 & 7.6497 \\
\hline Minimum: & $333.33 \mathrm{~ms}$ & $66.92 \mathrm{bpm}$ & Coet. of Variance. & $33.5693 \%$ & $46.6346 \%$ \\
\hline Max/Min: & 2.6900 & 26900 & Variance: & $42010.5849 \mathrm{mt} \mathrm{t}^{\mathrm{m}}$ & 2100.1200 tpmibpen \\
\hline Range: & $563.33 \mathrm{~ms}$ & $113.09 \mathrm{bpm}$ & Sid. Dev. (SDNN) & $204.9648 \mathrm{~ms}$ & $45.8271 \mathrm{bpm}$ \\
\hline Mean of NN: & $610.57 \mathrm{~ms}$ & $98.27 \mathrm{bpm}$ & Std Enr. [SE) & $11.5853 \mathrm{~ms}$ & $2.5903 \mathrm{bpm}$ \\
\hline Mean of dNN (MSD): & $48.9419 \mathrm{~ms}$ & $17.0533 \mathrm{bpm}$ & SDSD: & $79.1207 \mathrm{~ms}$ & $27.4312 \mathrm{bpm}$ \\
\hline Median: & $769.99 \mathrm{~ms}$ & $77.92 \mathrm{bom}$ & AMSSD: & $78.9939 \mathrm{~ms}$ & 27.3872 bpen \\
\hline $95 \%$ Conf. Intervat & $22.7072 \mathrm{~ms}$ & $5.0770 \mathrm{bpm}$ & NN50 Count: & 114 & 114 \\
\hline $99 \%$ Conf. Interval: & $29.8414 \mathrm{~ms}$ & $6.6721 \mathrm{bpm}$ & pNN50: & $36.4217 \%$ & $36.4217 \%$ \\
\hline
\end{tabular}

Figure 2. Linear time indexes of the subject at rest.

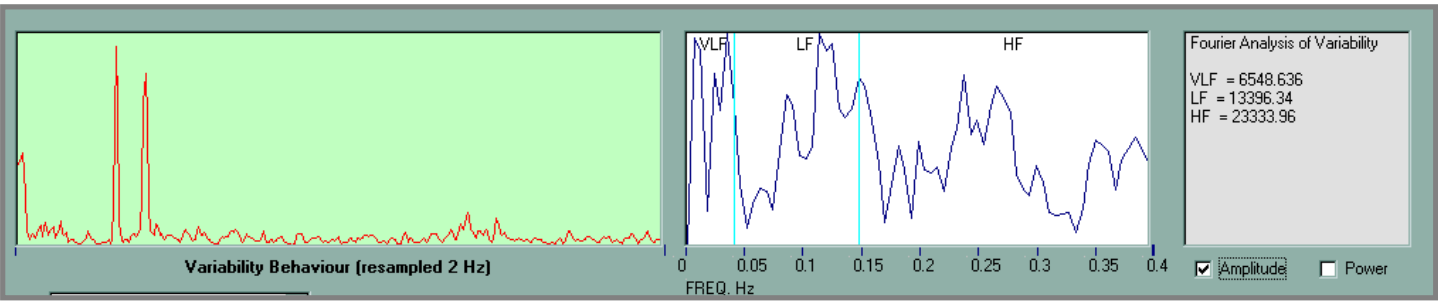

Figure 3. Time series of heart rhythm variability and values of variability (msec/Hz) in the case with absolute value (for details see [3]-[5]). The CZF method.

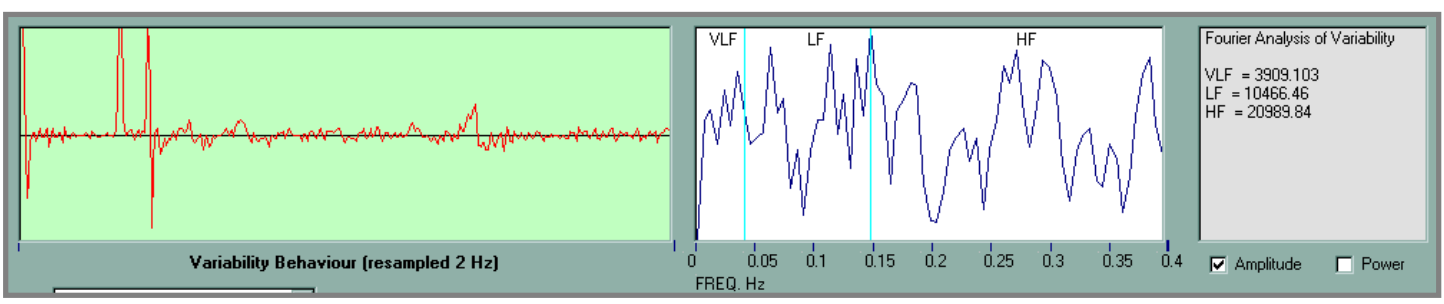

Figure 4. Time series of heart rhythm variability and values of variability (msec/Hz) in the case without absolute value (for details see [3]-[5]). The CZF method.

indication of the profound alterations that we have in this case for the subject before of the NPT treatment. The subsequent Poincaré plot in Figure 7, indicates the $R_{n} / R_{n+1}$ phase space distribution of the intervals. In normal condition we have the classical "cigar" distribution that is instead seriously altered in the case of the subject under investigation. The behaviour of the distribution, and the values of SD1, SD2 and ellipse area indicated that we were in presence of an atrial fibrillation. In Table 1 we have the results of the ApEN, SampEn, alpha 1 and alpha 2 of the Detrended Fluctuation Analysis. A tachogram of a normal subject usually gives values near to 1 as quantification of the correct variability and complexity of the R-R signal arising from the complex of the dynamics of the non linear ANS modulation. Increasing values of risk for the subject are evaluated in relation to values progressively fixed around 1 . In this case we have 0.194 for the ApEn and 0.127 for SampEn. These are 


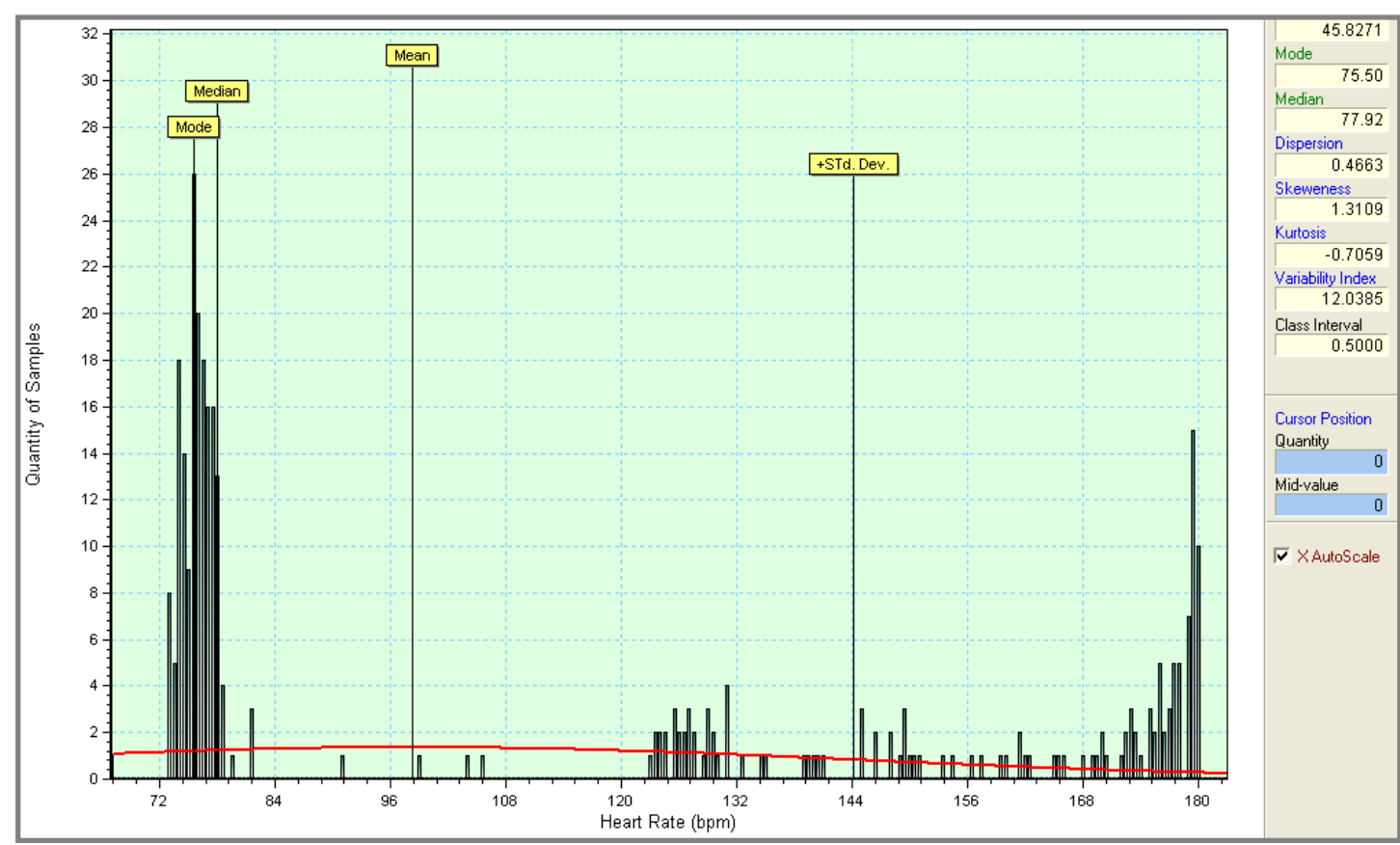

Figure 5. Histograms of HR for the subject at rest.

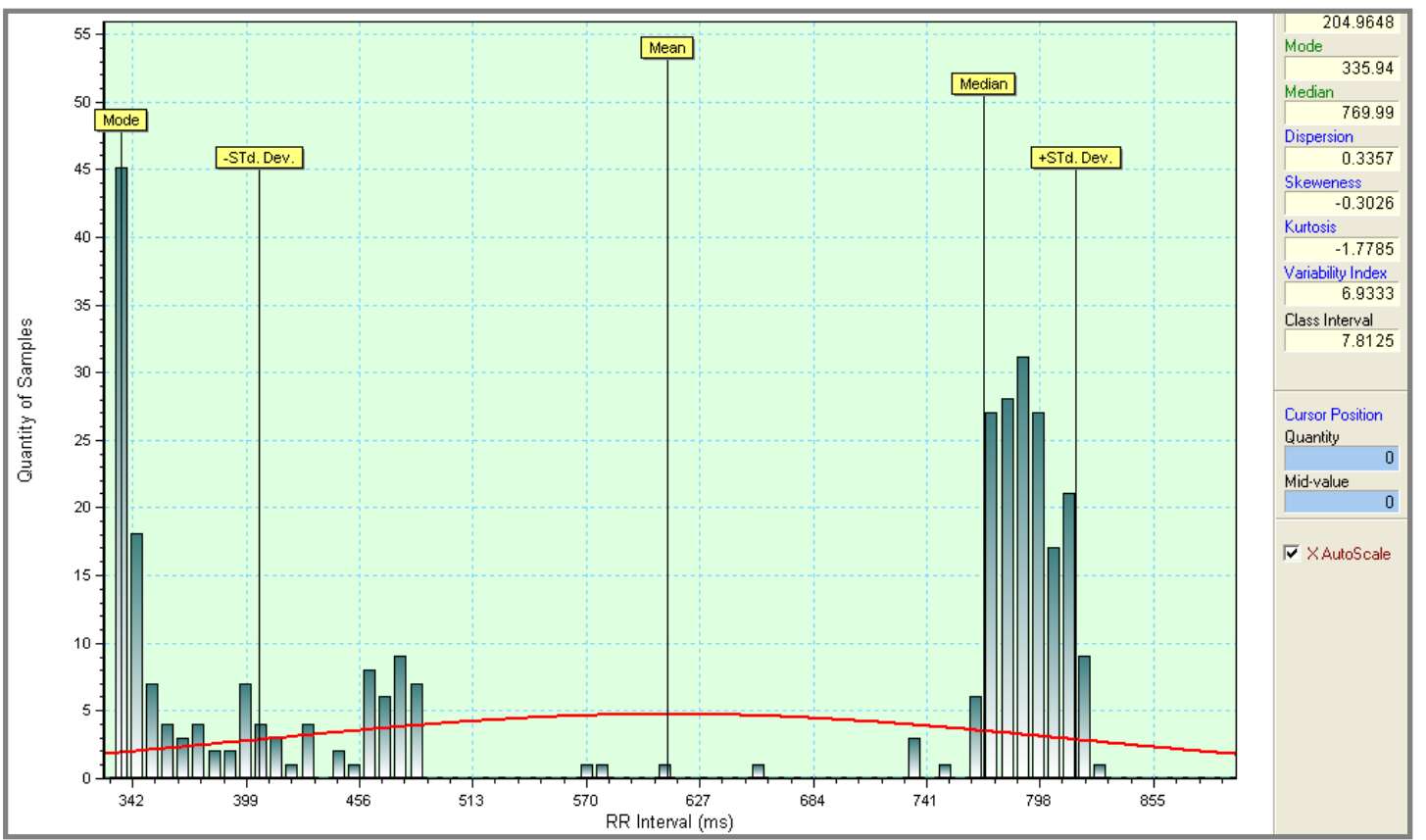

Figure 6. Histograms of R-R intervals for the subject at rest.

Table 1. Estimation of approximate entropy, of sample entropy, and detrended fluctuation analysis in the subject at rest.

$\begin{array}{cc}\text { Approximate entropy (ApEn) } & 0.194 \\ \text { Sample entropy (SampEn) } & 0.127 \\ \text { Detrended fluctuation (DFA): a1 } & 0.963 \\ \text { Detrended fluctuation (DFA): a2 } & 1.536\end{array}$


so serious violations of standard values to classify the subject at very high level of risk. However, the estimated values of alpha 1 and alpha 2 still indicate that the calculated values of the R-R intervals and of the tachogarm still maintain a sufficient correlation at short as well as long range. By comparison, in Figures 8-12 and Table 2

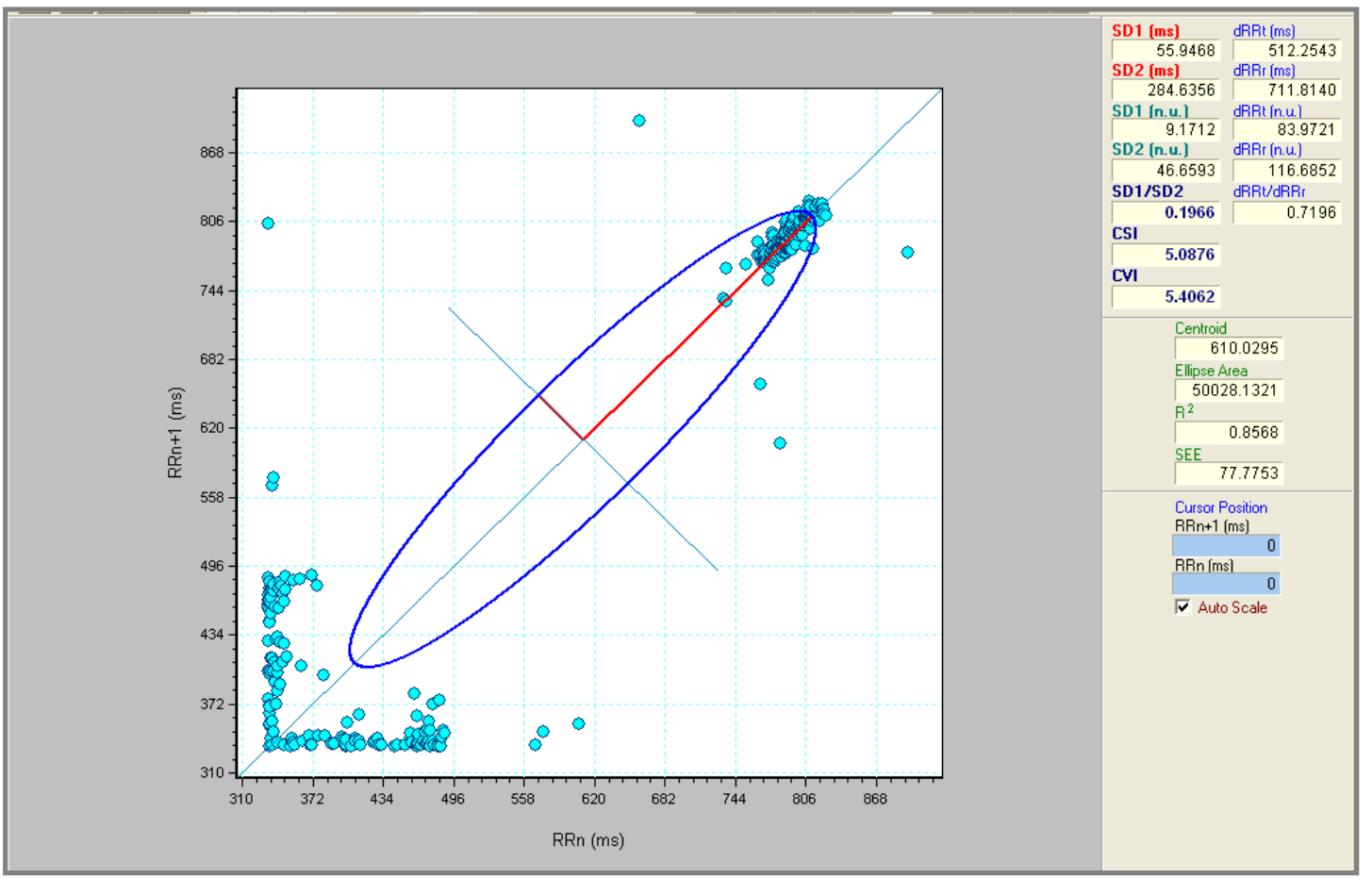

Figure 7. Poincaré plot relating the subject at rest.
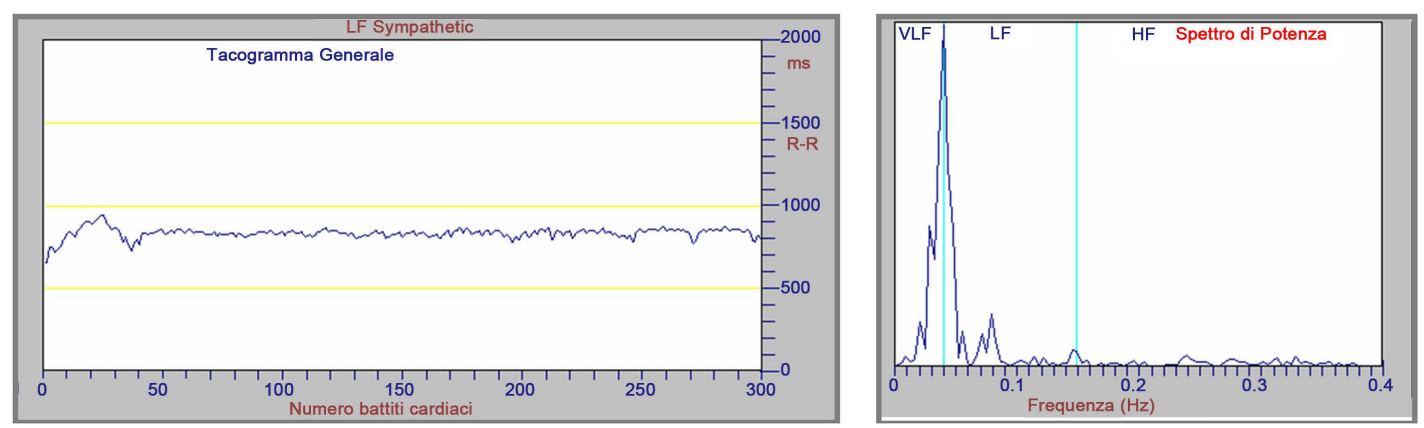

Figure 8. Tachogram obtained after ECG recording after the treatment PSD Spectrum $\left(\mathrm{msec}^{2} / \mathrm{Hz}\right)$ with three delineated bands, VLF, LF and HF.

\begin{tabular}{|c|c|c|c|c|c|}
\hline \multicolumn{6}{|c|}{ All Data } \\
\hline & RRI & $\mathrm{HR}$ & & RRI & $\mathrm{HR}$ \\
\hline Maximum: & $1243.00 \mathrm{~ms}$ & 135.44 bpm & InHRV: & 7.8592 & 3.7469 \\
\hline Minimum: & $443.00 \mathrm{~ms}$ & $48.27 \mathrm{bpm}$ & Coef. of Variance: & $6.0451 \%$ & $9.1343 \%$ \\
\hline Max./Min.: & 2.8059 & 2.8059 & Variance: & $2589.5140 \mathrm{~ms}^{x} \mathrm{~ms}$ & 42.3878 bpmbpm \\
\hline Range: & $800.00 \mathrm{~ms}$ & 87.17 bpm & Std. Dev. [SDNN]): & $50.8873 \mathrm{~ms}$ & $6.5106 \mathrm{bpm}$ \\
\hline Mean of NN: & $841.79 \mathrm{~ms}$ & $71.28 \mathrm{bpm}$ & Std. Err. (SE): & $3.0575 \mathrm{~ms}$ & $0.3912 \mathrm{bpm}$ \\
\hline Mean of dNN (MSD): & $16.5000 \mathrm{~ms}$ & $1.6264 \mathrm{bpm}$ & SDSD: & $62.1444 \mathrm{~ms}$ & 7.0684 bpm \\
\hline Median: & $843.00 \mathrm{~ms}$ & 71.17 bpm & RMSSD: & $62.0461 \mathrm{~ms}$ & 7.0591 bpm \\
\hline $95 \%$ Conf. Interval: & $5.9927 \mathrm{~ms}$ & $0.7667 \mathrm{bpm}$ & NN50 Count: & 6 & 6 \\
\hline $99 \%$ Conf. Interval: & $7.8756 \mathrm{~ms}$ & 1.0076 bpm & pNN50: & $2.1661 \%$ & $2.1661 \%$ \\
\hline
\end{tabular}

Figure 9. Values of linear time indexes in HRV analysis after the NPT treatment. 


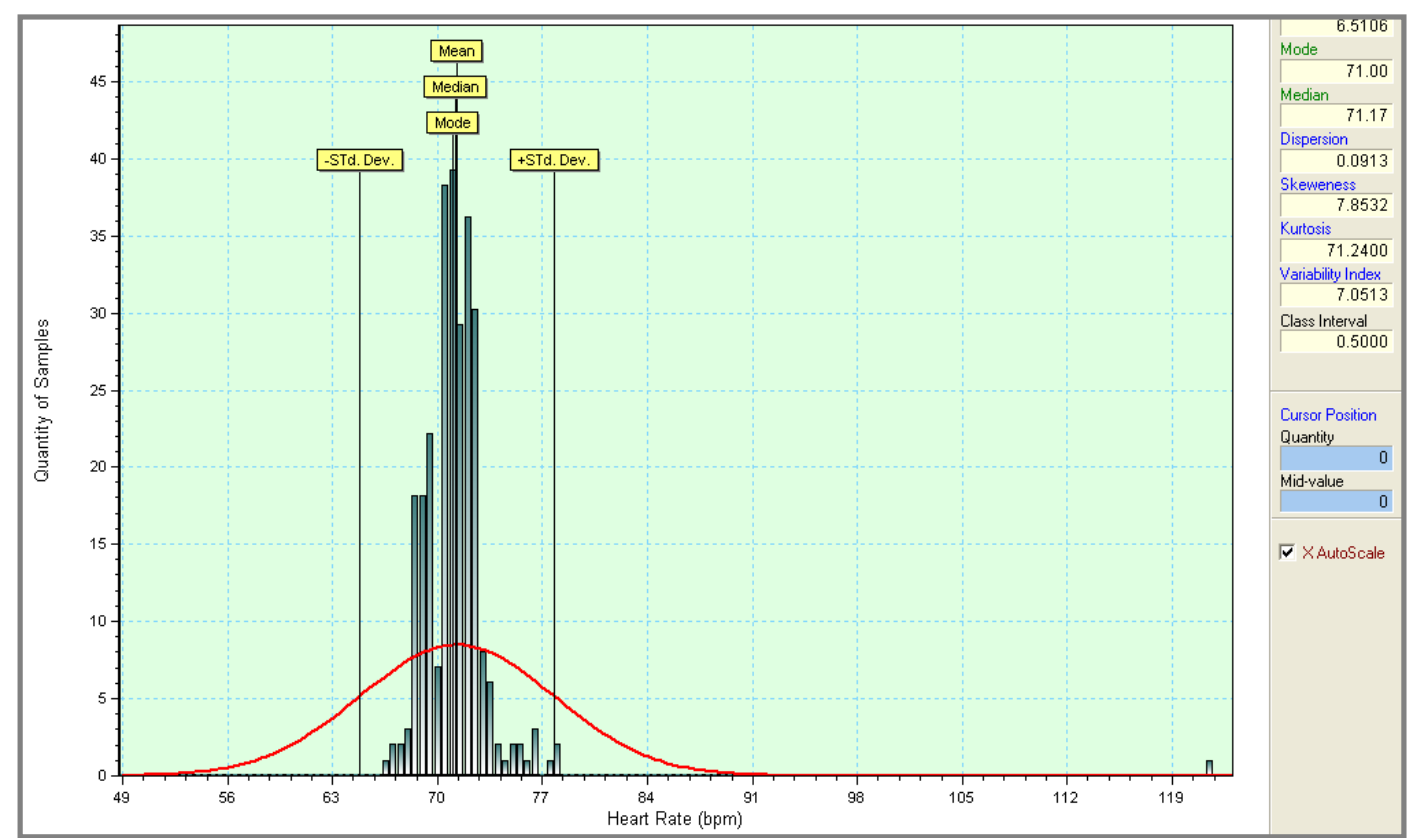

Figure 10. HR -Histogram distribution of the HR values after NPT treatment.

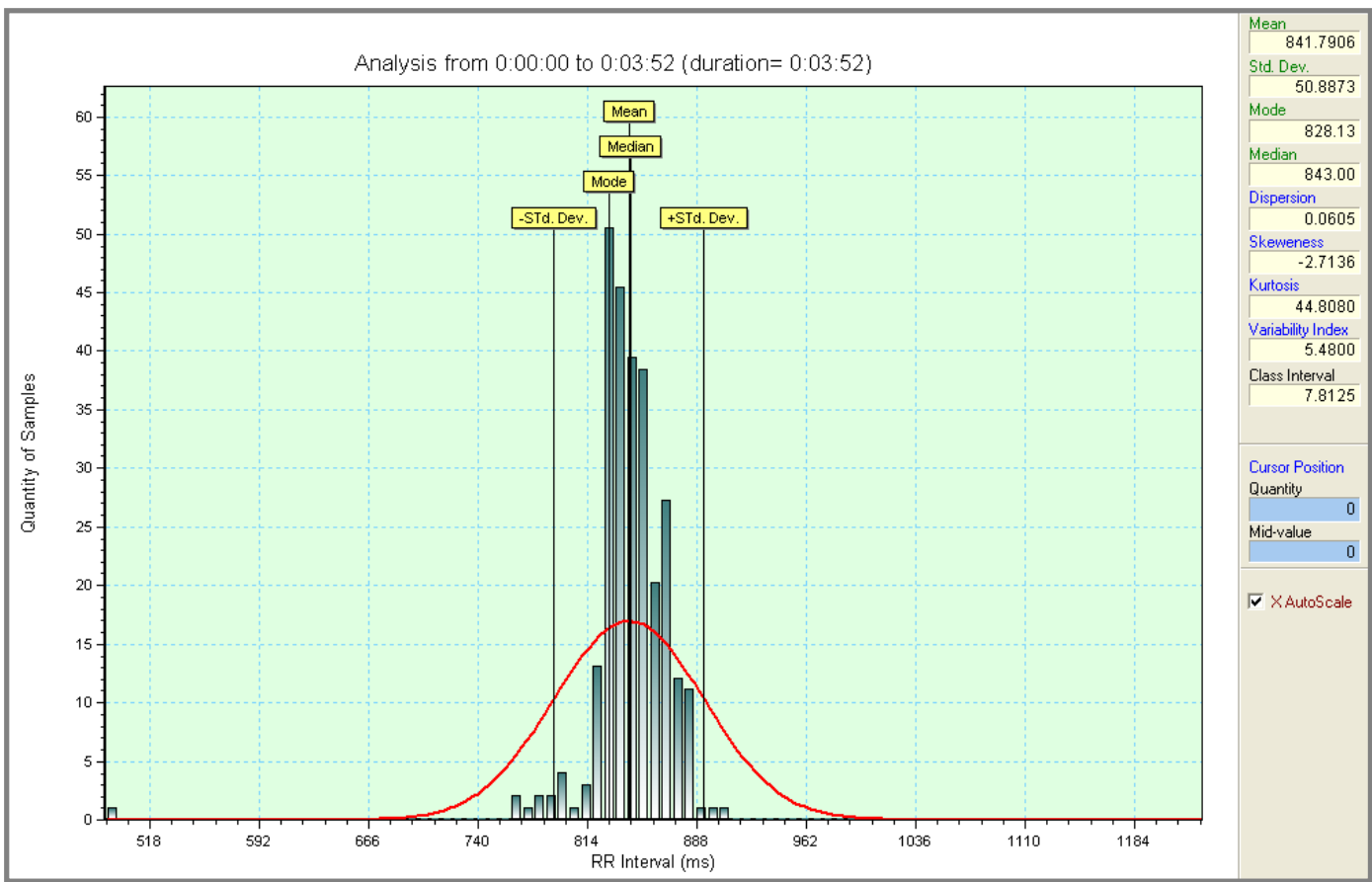

Figure 11. R-R Histogram distribution of the RR values after NPT treatment.

Table 2. Estimation of approximate entropy, of sample entropy, and detrended fluctuation analysis in the subject after the NPT treatment.

$\begin{array}{cc}\text { Approximate entropy (ApEn) } & 0.887 \\ \text { Sample Entropy (SampEn) } & 0.913 \\ \text { Detrended fluctuation (DFA): a1 } & 0.802 \\ \text { Detrended fluctuation (DFA): a2 } & 0.973\end{array}$




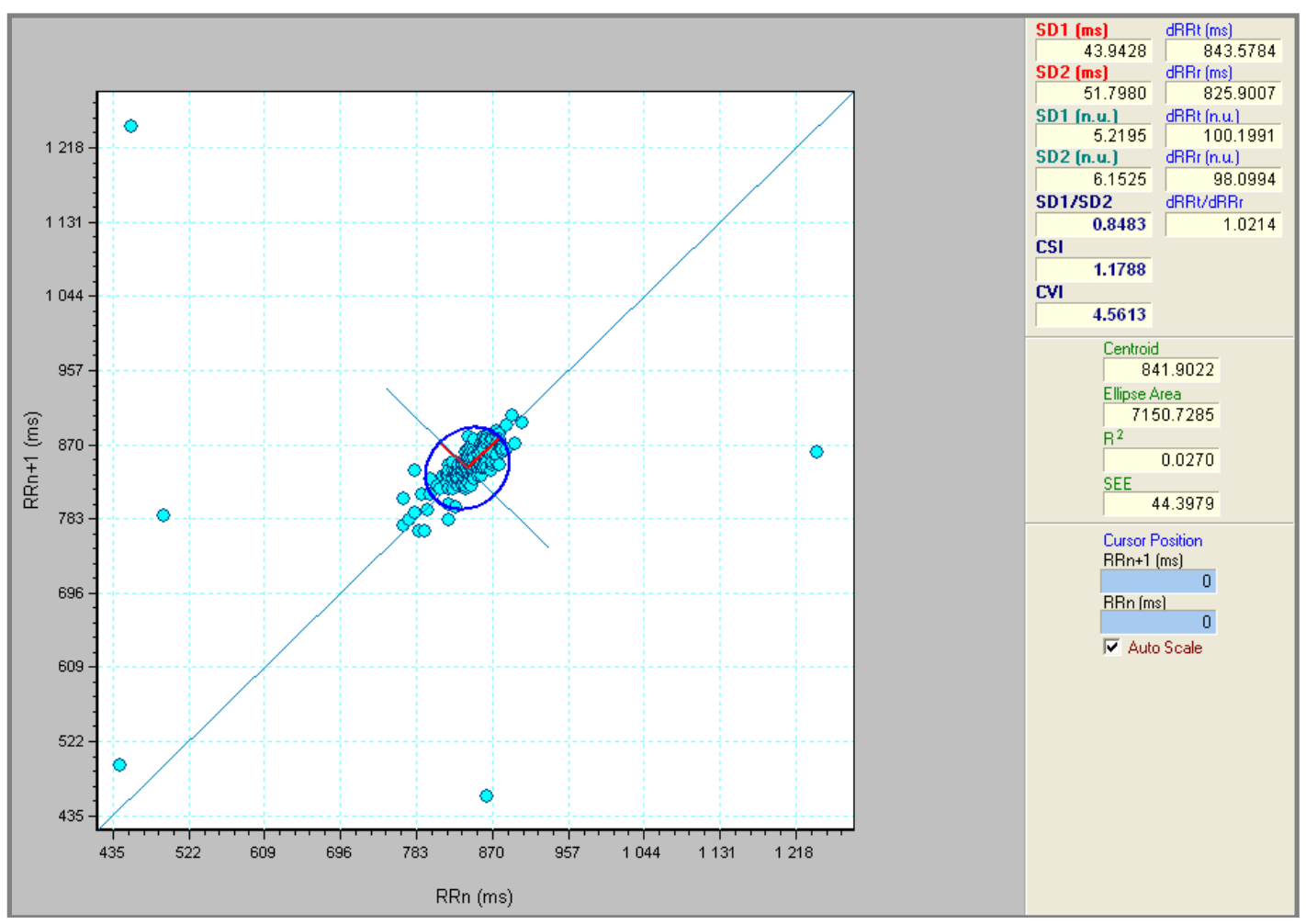

Figure 12. Poincaré plot of the subject after NPT treatment.

we have the values and graphs after the NPT treatment. The tachogram now represents a satisfactory behaviour with evident correct alternations. The values in the VLF, LF and HF bands now are re-entered rather in a satisfactory manner near the normal ranges. The histograms of the HR and R-R intervals reveal the normal statistical distribution; the Poincaré plot evidences now the typical "cigar” distribution also if still rather contracted. Atrial fibrillation has been overcome. A net improvement in the values of ApEN and SampEn has been reached leading the subject out of the previous serious risk.

\section{References}

[1] Tsuji, H., Larson, M.G., Venditti Jr., F.J., Manders, E.S., Evans, J.C. and Feldman, C.L. (1996) Impact of Reduced Heart Rate Variability on Risk for Cardiac Events. The Framingham Heart Study. Circulation, 94, 2850-2855. http://dx.doi.org/10.1161/01.CIR.94.11.2850

[2] Stein, P.K. and Kleiger, R.E. (1999) Insights from the Study of Heart Rate Variability. Annual Review of Medicine, 50, 249-261. http://dx.doi.org/10.1146/annurev.med.50.1.249

[3] Conte, E., Giroldini, W., Laterza, V., Conte, S., Pieralice, M., Casciaro, F., De Masi De Luca, G., Giuliano, A.F.M., Mendolicchio, L. and Todarello, O. (2014) Experimental Results on a New Method for Analysis of Heart Rate Variability. World Journal of Cardiovascular Diseases, 4, 385-389. http://dx.doi.org/10.4236/wjcd.2014.48048

[4] Conte, E., Federici, A. and Zbilut, J.P. (2009) A New Method Based on Fractal Variance Function for Analysis and Quantification of Sympathetic and Vagal Activity in Variability of R-R Time Series in ECG Signals. Chaos, Solitons and Fractals, 41, 1416-1426. http://dx.doi.org/10.1016/j.chaos.2008.05.025

[5] Conte, E. (2014) A New Method for Analysis of Heart Rate Variability, Asymmetry and BRS. Chaos and Complexity Letters, 8, 8-15.

[6] McNally, E.M. and Sparano, D. (2011) Mechanisms and Management of the Heart in Myotonic Dystrophy. Heart, 97, 1094-1100. http://dx.doi.org/10.1136/hrt.2010.214197

[7] Groh, W.J., Groh, M.R., Saha, C., Kincaid, J.C., Simmons, Z. and Ciofaloni, E. (2008) Electrocardiographic Abnormalities and Sudden Death in Myotonic Dystrophy Type 1. New England Journal of Medicine, 358, 2688-2697. http://dx.doi.org/10.1056/NEJMoa062800

[8] Olofsson, B.O., Niklasson, U., Forsberg, H., Bjerle, P., Anderson, S. and Henriksson, A. (1990) Assessment of Auto- 
nomic Nerve Function in Myotonic Dystrophy. Journal of the Autonomic Nervous System, 29, 187-192. http://dx.doi.org/10.1016/0165-1838(90)90144-8

[9] den Heijer, J.C., van Dijk, J.G., Bollen, W.L., Bos, J.E. and Wintzen, A.R. (1991) Assessment of Autonomic Function in Myotonic Dystrophy. Journal of Neurology, Neurosurgery Psychiatry, 54, 531-534. http://dx.doi.org/10.1136/jnnp.54.6.531

[10] Inoue, K., Ogata, H., Matsui, M., Hayano, J., Miyake, S., Kumashiro, M., et al. (1995) Assessment of Autonomic Function in Myotonic Dystrophy by Spectral Analysis of Heart-Rate Variability. Journal of the Autonomic Nervous System, 55, 131-134. http://dx.doi.org/10.1016/0165-1838(95)00040-5

[11] Flachenecker, P., Schneider, C., Cursiefen, S., Ricker, K., Toyka, K.V. and Reiners, K. (2003) Assessment of Cardiovascular Autonomic Function in Myotonic Dystrophy Type 2 (DM2/PROMM). Neuromuscular Disorders, 13, 289293. http://dx.doi.org/10.1016/S0960-8966(02)00277-8

[12] Hardin, B.A., Lowe, M.R., Bhakta, D. and Groh, W.J. (2003) Heart Rate Variability Declines with Increasing Age and CTG Repeat Length in Patients with Myotonic Dystrophy Type 1. Annals of Noninvasive Electrocardiology, 8, 227232. http://dx.doi.org/10.1046/j.1542-474X.2003.08310.x

[13] Di Leo, R., Rodolico, C., De Gregorio, C., Recupero, A., Cogliatore, S. and Annesi, G. (2004) Cardiovascular Autonomic Control in Myotonic Dystrophy Type 1: A Correlative Study with Clinical and Genetic Data. Neuromuscular Disorders, 14, 136-141. http://dx.doi.org/10.1016/j.nmd.2003.11.002

[14] Rakocević-Stojanović, V., Milovanović, B., Ivić, N., Ille, T., Marjanovic, I., Stevic, Z., et al. (2007) Cardiac Autonomic Nervous System in Patients with Myotonic Dystrophy Type 1. Acta Myologica, 26, 112-114.

[15] Ware, K., Conte, E., Marvulli, R., Ianieri, G., Megna, M., Pierangeli, E., Conte, S., Mendolicchio, L. and Pellegrino, F. (2015) Case Report: Generalized Mutual Information (GMI) Analysis of Sensory Motor Rhythm in a Subject Affected by Facioscapulohumeral Muscular Dystrophy after Ken Ware Treatment. World Journal of Neuroscience, 5, 67-81. http://dx.doi.org/10.4236/wjns.2015.52008

[16] Ware, K., Conte, E.., Marvulli, R., Ianieri, G., Megna, M., Pierangeli, E., Conte, S., Mendolicchio, L. and Pellegrino, F. (2015) Analysis of the Autonomic Regulation in a Case of Facioscapulohumeral Muscular Dystrophy after Ken Ware Treatment. World Journal of Neuroscience, 5, 162-173. http://dx.doi.org/10.4236/wjns.2015.52018

[17] Task Force of the European Society of Cardiology and the North American Society of Pacing and Electrophysiology (1996) Heart Rate Variability. Standards of Measurement, Physiologic Interpretation, and Clinical Use. Circulation, 93, 1043-1065. http://dx.doi.org/10.1161/01.CIR.93.5.1043 